Bulgakova, M. 310

English, A.W. 298

Falcon, I. 336

Gregor, R.J. 310

Hodson-Tole, E.F. 310

Lewek, M.D. 290

Maas, H. 310

McCulloch, K. 290

Mercer, V.S. 290
Nichols, T.R. 325,336

Nicolini, J. 298

Prilutsky, B.I. 310

Sabatier, M.J. 298

Segal, R.L. 289, 290

Stahl, V.A. 325, 336

To, B.N. 298

\title{
Subject Index Vol. 193, No. 5, 2011
}

Crural fascia 325

Joint moment 310

- power 310

Locomotion 298, 310, 325, 336

Motor compensation 310

Nerve injury 310

Peripheral nerve injury 290

Physical therapy 290
Popliteal fat pad 336

Proprioception 290

Rehabilitation 290

Sciatic nerve 298

Soleus 298

Tibialis anterior 298

Translation 290

Triceps surae 325 